\title{
A simple model for squirt-flow dispersion and attenuation in fluid-saturated granular rocks
}

\author{
Boris Gurevich ${ }^{1,2}$, Dina Makarynska ${ }^{1}$, Osni Bastos de Paula ${ }^{1,3}$, and Marina Pervukhina ${ }^{2}$
}

\begin{abstract}
A major cause of seismic attenuation in fluid-saturated rocks is the flow of the pore fluid induced by the passing wave. At sonic and ultrasonic frequencies, attenuation appears to be dominated by the local (pore-scale) flow between pores of different shapes and orientations. A simple squirt flow model is developed in which all of the parameters can be independently measured or estimated from measurements. The pore space of the rock is assumed to consist of stiff porosity and compliant (or soft) pores present at grain contacts. The effect of isotropically distributed compliant pores is modeled by considering pressure relaxation in a disk-shaped gap between adjacent grains. This derivation gives the complex and frequency-dependent effective bulk and shear moduli of a rock, in which the compliant pores are liquid saturated and stiff pores are dry. The resulting squirt model is consistent with Gassmann's and Mavko-Jizba equations at low and high frequencies, respectively. The magnitude of attenuation and dispersion given by the model is directly related to the variation of dry bulk modulus with pressure and is relatively independent of fluid properties.
\end{abstract}

\section{INTRODUCTION}

A major cause of elastic wave attenuation in fluid-saturated rocks is the flow of the pore fluid induced by the passing wave. When an elastic wave propagates through a fluid-saturated medium, it creates local pressure gradients within the fluid phase, resulting in fluid flow and corresponding internal friction until the pore pressure is equilibrated. The fluid flow can take place on various length scales.

Flow between mesoscopic (larger than the pore size but smaller than the wavelength) patches of rock with different stiffness due to rock heterogeneity (White et al., 1975; Pride et al., 2003) or spatial variations in fluid saturation (White, 1975; Gist, 1994; Toms et al., 2007) is believed to be significant at seismic frequencies. At sonic and ultrasonic frequencies, attenuation appears to be dominated by the local (pore-scale) flow between pores of different shapes and orientations (Mavko and Nur, 1975, 1979; Jones, 1986). Mesoscopic flow can be treated using all of the machinery of Biot's theory of poroelasticity (Biot, 1956a, b, 1962; Bourbié et al., 1987) with spatially varying coefficients (Dutta and Ode, 1979a, b; Lopatnikov and Gurevich, 1988; Lopatnikov et al., 1990; Auriault and Boutin, 1994; Gurevich and Lopatnikov, 1995; Pride et al., 2004; Müller and Gurevich, 2005; Johnson, 2001).

Modeling local flow, also known as squirt, cannot be done in a similar manner because local flow depends on various parameters describing pore shapes and orientations. Most theoretical models of squirt-flow attenuation are based on the analysis of aspect ratio distributions (Mavko and Nur, 1979; O'Connell and Budiansky, 1977; Palmer and Traviolia, 1980); a comprehensive review of these earlier studies is by Jones (1986). An alternative approach is based on the recognition that the pore space of many rocks has a binary structure (Walsh, 1965; Mavko and Jizba, 1991; Shapiro, 2003): relatively stiff pores, which form most of the pore space, and relatively compliant (or soft) pores, which are responsible for the pressure dependency of the elastic moduli (Murphy et al., 1986; Dvorkin et al., 1995; Chapman et al., 2002). In particular, Dvorkin et al. (1995) model a rock as a granular aggregate in which the grains themselves are assumed porous. Intergranular pores are stiff, whereas the intragranular micropores are soft. This model was later reformulated and refined by Pride et al. (2004). The advantage of the porous grain model, particularly in the formulation of Pride et al. (2004) over all other squirt models is in the fact that the medium can be treated as poroelastic on the subpore scale and thus is amenable to treatment using Biot's equations of poroelasticity with spatially varying coefficients (Pride and Berryman, 2003). This model is also consistent with Mavko and Jizba's (1991) predictions for the high-frequency

Manuscript received by the Editor 9 February 2010; published online 8 December 2010.

${ }^{1}$ Curtin University of Technology, Department of Exploration Geophysics, Perth, Australia. E-mail: B.Gurevich@curtin.edu.au and D.Makarynska@ curtin.edu.au.

${ }^{2}$ CSIRO Earth Sciences and Resource Engineering, Kensington, Australia. E-mail: Marina.Pervukhina@ csiro.au.

${ }^{3}$ PETROBRAS, Rio de Janeiro, Brazil.E-mail: osnidepaula@ petrobras.com.br.

(C) 2010 Society of Exploration Geophysicists. All rights reserved. 
limit of elastic moduli, which are known to be in good agreement with laboratory measurements (Mavko and Jizba, 1994; Endres and Knight, 1997; Wulff and Burkhardt, 1997). However, the concept of porous grains is somewhat abstract, and interpretation of parameters of this imaginary microporous grain in terms of rock properties is difficult. Furthermore, application of Biot's theory to microporous grains assumes that compliant pores are small compared with the grain size; this may not be the case for real rocks.

An appealing alternative is the approach of Murphy et al. (1986), who consider compliant pores as gaps at contacts between adjacent grains, see also Mayr and Burkhardt (2006). However, the model of Murphy et al. (1986) is not consistent with the well-established highfrequency predictions of Mavko and Jizba (1991); in fact, its highfrequency prediction for the elastic moduli is unrealistically high. This inconsistency stems from the fact that the particular formulation of Murphy et al. (1986) is developed within the framework of the Hertz-Mindlin grain contact theory (Digby, 1982; Winkler, 1983), in which grains themselves are assumed rigid and the compliance of the rock is caused solely by weak grain contacts. In the highfrequency limit, fluid pressure cannot relax between the intergranular gap and the surrounding (stiff) pore, making its compliance vanishingly small and rock unrealistically stiff.

In this paper, we propose a new model of squirt-flow attenuation that uses a pressure relaxation approach of Murphy et al. (1986) in conjunction with the discontinuity tensor formulation of Sayers and Kachanov (1995). The resulting model is consistent with the Gassmann (1951) and Mavko-Jizba equations at low and high frequencies, respectively, and with the piezosensitivity model of Shapiro (2003). It can also be naturally incorporated into Biot's theory of poroelasticity to obtain velocity and attenuation prediction in a broad frequency range.

The paper is organized as follows. First, we develop a theoretical model for the frequency dependence of elastic moduli due to pressure relaxation in the intergranular contact area. We then analyze the asymptotic behavior of this model in several limiting cases and present a simplified formulation for the most important case of liquid saturation. Finally, we illustrate model prediction with a few laboratory data examples.

\section{THEORETICAL MODEL}

Following Walsh (1965), Mavko and Jizba (1991), and Shapiro (2003), we assume that the pore space of the rock consists of stiff and compliant pores, which form fully interconnected pore space. We assume that the dry rock frame (skeleton) is homogeneous (i.e., consists of a single isotropic mineral with bulk modulus $K_{g}$ and shear modulus $\mu_{g}$ ). The frame is also assumed isotropic on microscale (pore scale) and macroscale (wavelength scale) and is characterized by stiff porosity $\phi_{s}$, compliant porosity $\phi_{c} \ll \phi_{s}$, total porosity $\phi$ $=\phi_{s}+\phi_{c} \approx \phi_{s}$, permeability $\kappa$, and bulk and shear moduli $K_{\mathrm{dry}}$ and $\mu_{\mathrm{dry}}$, respectively.

The aim of this section is to derive expressions for frequency-dependent moduli of our rock when it is fully saturated by a single fluid with a bulk modulus $K_{f}$ and dynamic viscosity $\eta$. The frequency dependency (dispersion) in our rock can be caused by two principal mechanisms: global flow dispersion due to the flow of fluid relative to the solid frame caused by the pressure gradients between peaks and troughs of the wave, and squirt flow between compliant pores and stiff pores. In this paper, we are principally concerned with squirt flow. Therefore, to ensure that Biot's dispersion is negligible, we will, for the time being, assume that the characteristic frequency $f_{\text {Biot }}$ of Biot's dispersion is much higher than the squirt characteristic frequency $f_{c}$ and the frequency of the propagating wave $f$. Both of these conditions will later be lifted.

\section{Low-frequency (relaxed) moduli}

In the low-frequency limit, the bulk and shear moduli of our fluidsaturated rock are given by Gassmann's equations (Gassmann, 1951; White, 1983)

$$
\frac{1}{K_{\mathrm{low}}}=\frac{1}{K_{g}}+\frac{\phi\left(\frac{1}{K_{f}}-\frac{1}{K_{g}}\right)}{1+\phi\left(\frac{1}{K_{f}}-\frac{1}{K_{g}}\right) /\left(\frac{1}{K_{\mathrm{dry}}}-\frac{1}{K_{g}}\right)}
$$

and

$$
\mu_{\mathrm{low}}=\mu_{\mathrm{dry}} .
$$

Gassmann's equations 1 and 2 are valid when $f \ll f_{c}$. Physically, this means that the wave frequency is sufficiently low so that fluid pressure has enough time to equilibrate between stiff and compliant pores during half-wave cycle. Thus, the moduli given by Gassmann's equations 1 and 2 can be called "relaxed moduli."

\section{High-frequency (unrelaxed) moduli}

When the frequency is higher than the squirt characteristic frequency, $f \gg f_{c}$, then the fluid pressure does not have enough time to equilibrate between stiff and compliant pores during half-wave cycle (so-called unrelaxed state). Then, compliant pores at the grain contacts are effectively isolated from the stiff pores and hence become stiffer with respect to normal (but not tangential) deformation. To quantify this effect, Mavko and Jizba (1991) considered the socalled modified frame - the rock in which only compliant pores are filled with the fluid, whereas stiff pores are empty — and showed that unrelaxed (high-frequency) bulk and shear moduli, $K_{u f}$ and $\mu_{u f}$, respectively, of this modified frame are given by

$$
\frac{1}{K_{u f}(P)} \approx \frac{1}{K_{h}}+\left(\frac{1}{K_{f}}-\frac{1}{K_{g}}\right) \phi_{c}(P)
$$

and

$$
\frac{1}{\mu_{u f}(P)} \approx \frac{1}{\mu_{\mathrm{dry}}(P)}-\frac{4}{15}\left(\frac{1}{K_{\mathrm{dry}}(P)}-\frac{1}{K_{u f}(P)}\right),
$$

where $K_{h}$ is the dry bulk modulus of a hypothetical rock without the compliant porosity (see also Berryman, 2007) and $P$ is differential pressure. Note that for most rocks, $\phi_{c}(P)$ is on the order of $10^{-3}$ or smaller (Mavko and Jizba, 1991; Shapiro, 2003). For typical reservoir liquids (not gases!), the bulk modulus $K_{f}$ is on the order of one tenth of the dry rock modulus and is much smaller than the mineral modulus $K_{g}$. Therefore, the second term in the right-hand side of equation 3 is at most on the order of 0.01 of the first term and hence is negligible for most liquids. This means that the bulk modulus of the modified frame is almost independent of the pressure. However, the shear modulus (and hence, compressional and shear velocities) still depends on pressure through the pressure dependency of the dry bulk and shear moduli $K_{\mathrm{dry}}(P)$ and $\mu_{\mathrm{dry}}(P)$. 
Equation 3 for the unrelaxed bulk modulus of the modified frame was derived by Mavko and Jizba (1991) as a first-order expansion in the powers of complaint porosity and implies that $\phi_{c}\left(K_{f}^{-1}\right.$ $\left.-K_{g}^{-1}\right) \ll K_{h}^{-1}$. Indeed, as discussed above, if the saturating fluid is liquid, this condition is usually satisfied. However, as will be seen later in our derivation, this condition is too restrictive for our purposes. A more general expression for the unrelaxed frame modulus (without any restriction on the fluid compressibility) can be derived using Sayers-Kachanov discontinuity formalism (Sayers and Kachanov, 1995; Sayers and Han, 2002). The detailed derivation is given in Gurevich et al. (2009a). The result reads

$$
\frac{1}{K_{u f}(P)}=\frac{1}{K_{h}}+\frac{1}{\frac{1}{\frac{1}{K_{\text {dry }}(P)}-\frac{1}{K_{h}}}+\frac{1}{\left(\frac{1}{K_{f}}-\frac{1}{K_{g}}\right) \phi_{c}(P)}} .
$$

When the fluid is liquid, the term $\left(K_{\mathrm{dry}}^{-1}-K_{h}^{-1}\right)^{-1}$ can be neglected compared with the term $\left[\phi_{c}\left(K_{f}^{-1}-K_{g}^{-1}\right)\right]^{-1}$, and equation 5 yields Mavko-Jizba equation 3. However, equation 5 is more general in that it is free of any restriction on the fluid modulus. In particular, for a dry rock $K_{f}=0$, the term $\phi_{c}\left(K_{f}^{-1}-K_{g}^{-1}\right)^{-1}$ vanishes and $K_{u f}$ reduces to the dry modulus $K_{\text {dry }}$, as it should.

The fully saturated unrelaxed moduli can be obtained from the modified frame moduli using Gassmann's equations

$$
\frac{1}{K_{\text {high }}(P)}=\frac{1}{K_{g}}+\frac{\phi_{s}\left(\frac{1}{K_{f}}-\frac{1}{K_{g}}\right)}{1+\phi_{s}\left(\frac{1}{K_{f}}-\frac{1}{K_{g}}\right) /\left(\frac{1}{K_{u f}(P)}-\frac{1}{K_{g}}\right)}
$$

and

$$
\mu_{\text {high }}(P)=\mu_{u f}(P) \text {. }
$$

The use of Gassmann's equations for high frequencies may raise some questions. Indeed, Gassmann's equations assume that fluid pressure is equalized within the representative volume (RV) of the pore space. Pressure equilibration can be achieved if the pore space is interconnected and the frequency is sufficiently low to allow enough time for pressure to equilibrate within the wave's half-cycle. As previously discussed, higher frequency may prevent equilibration of pressure within a half-wave cycle, effectively making the pores hydraulically isolated. However, the condition of interconnected pore space is not a necessary condition for the validity of Gassmann's or Biot's equations. The key condition is the spatially uniform fluid pressure in the pores (within RV). In particular, Gassmann's equations are exact for a material with a dilute concentration of randomly distributed isolated spherical pores because the induced pressure is the same in all of these pores (for any frequency below the characteristic frequency of scattering). Approximately, this is also true for all "equant" pores (pores with aspect ratio on the order $O(1)$ ) (Thomsen, 1985). Recently, Grechka (2009) showed numerically that Gassmann's equations are excellent approximations for isolated pores of aspect ratio larger than 0.1 . Therefore, as suggested by Mavko and Jizba (1991), Gassmann's and Biot's equations are applicable to the stiff pores of our system at seismic and ul- trasonic frequencies. This is also consistent with the well-established observation that the squirt-flow dispersion between seismic and ultrasonic frequencies is caused mainly by compliant porosity and is negligible at high effective stress, at which compliant porosity is mostly closed and only stiff pores remain. Indeed, the characteristic frequency of squirt-flow dispersion is usually written as

$$
f_{c}=\alpha^{3} \frac{K}{\eta}
$$

where $\alpha$ is the mean aspect ratio of the pores and $K$ is the rock's bulk modulus (Jones, 1986). For stiff pores $\alpha=O(1)$; thus, the squirt frequency is much larger than $1 \mathrm{MHz}$ even for fluids 1000 times more viscous than water.

To summarize, the high-frequency moduli of the fully saturated rock are given by Gassmann's equations 6 and 7 with the modified frame moduli given by equations 4 and 5 .

\section{Frequency-dependent (partially relaxed) moduli}

\section{Modified frame}

Now we set out to derive expressions for the moduli of the rock at intermediate frequencies, which represent some intermediate state between the low- and high-frequency limits. Note that because compliant porosity is always small, $\phi_{c} \ll \phi_{s}$, we have $\phi_{s} \approx \phi$. Therefore, saturated high-frequency moduli are given by the same Gassmann's equations 6 and 7 as the low-frequency moduli (equations 1 and 2), except that for high frequencies the dry moduli $K_{\text {dry }}$ and $\mu_{\text {dry }}$ are replaced by the unrelaxed frame moduli $K_{u f}$ and $\mu_{u f}$. Furthermore, we note that the dry moduli $K_{\text {dry }}$ and $\mu_{\text {dry }}$ can also be considered as the moduli of the modified frame (rock with empty stiff pores but fluidfilled compliant pores) but in a relaxed state; that is, when compliant pores are in full-pressure equilibrium with the stiff pores. Because in the modified frame stiff pores are empty, the pressure in compliant pores is zero. Thus, the relaxed moduli of the modified frame are equal to the rock's dry, or drained, moduli. In other words, the fully saturated (undrained) moduli of the rock in the low- and high-frequency limits are given by the same Gassmann's equations

$$
\begin{aligned}
& \frac{1}{K_{\mathrm{sat}}(P, \omega)} \\
& \quad=\frac{1}{K_{g}}+\frac{\phi_{s}\left(\frac{1}{K_{f}}-\frac{1}{K_{g}}\right)}{1+\phi_{s}\left(\frac{1}{K_{f}}-\frac{1}{K_{g}}\right) /\left(\frac{1}{K_{m f}(P, \omega)}-\frac{1}{K_{g}}\right)}
\end{aligned}
$$

and

$$
\mu_{\mathrm{sat}}(P, \omega)=\mu_{m f}(P, \omega),
$$

where the modified frame moduli $K_{m f}$ and $\mu_{m f}$ are to be taken at lowand high-frequency limits, respectively. Furthermore, it is logical to assume that moduli at the intermediate frequencies between these limits are also given by Gassmann's equations 9 and 10 with the modified $K_{m f}$ and $\mu_{m f}$ taken at the corresponding frequency. Thus, the problem of finding the frequency-dependent moduli of the fully saturated rock reduces to the problem of finding the frequency de- 
pendency of the moduli of the modified frame, where compliant pores are fluid filled and stiff pores are dry.

\section{Fluid relaxation in the area of grain contact}

It is often the case with heterogeneity-related dispersion mechanisms that the low- and high-frequency limits of elastic moduli are independent of intricacies of geometry, but the shape of the frequency dependency of the moduli is defined by the particular geometrical configuration. A good example is a porous rock saturated with patches of two immiscible fluids (Johnson, 2001) in which the low- and high-frequency moduli are uniquely defined by the properties of the rock matrix, the properties of the two fluids, and their volume fractions. In contrast, the frequency dependency of the moduli (and attenuation) is controlled by the size and shape of the patches.

The squirt-flow dispersion has the same feature. The low-frequency moduli are given by the exact Gassmann's equations, which involve only one explicit parameter of the pore space-total porosity. The high-frequency limit (ignoring Biot's dispersion for a moment) as given by equations 4-7 requires, additionally, the knowledge of the compliant porosity. However, to model the frequency dependency of the moduli, we need to assume a particular geometrical configuration. Here we assume a particular geometry proposed by Murphy et al. (1986): a compliant pore forms a disk-shaped gap between two adjacent grains, and its edge opens into a toroidal stiff pore (Figure 1). It is assumed that the gap also has asperities; thus, its stiffness is finite even when the gap is empty. However, these asperities are assumed (somewhat arbitrarily) not to affect the geometry of the gap as far as the fluid movement is concerned. The gap has radius $a$ and thickness $h$. The additional effective stiffness $K^{*}$ of the gap due to the presence of fluid can be defined as a ratio of the force $\Delta F$ (acoustic force) exerted by the fluid onto the gap wall to the uniaxial dynamic loading (displacement) $-\Delta h$

$$
K^{*}=\frac{\Delta F}{-\Delta h} .
$$

The force is essentially the integral of pressure over the surface $S_{\mathrm{g}}$ of the gap,

$$
\Delta F=\int_{S_{g}} p(r) d S
$$

For sinusoidal loading, $\Delta h \exp (i \omega t)$ with frequency $\omega=2 \pi f$, fluid pressure $p$ can be obtained as a solution of the ordinary differential equation

$$
\frac{d^{2} p}{d r^{2}}+\frac{1}{r} \frac{d p}{d r}+k^{2} p=C
$$

where $r$ is the radial coordinate;

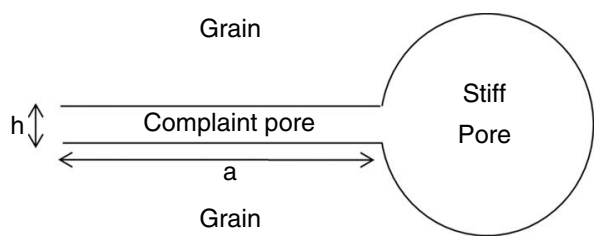

Figure 1. Sketch of the model configuration (Murphy et al., 1986). Soft pore forms a disc-shaped gap between two grains, and its edge opens into a toroidal stiff pore.

$$
k^{2}=\frac{-i \omega h_{0} D}{K_{f}},
$$

is the wavenumber of the pressure diffusion wave in the gap; $D$ $=12 \eta / h_{0}^{3}$ is the viscous resistance, $C=i \omega D \Delta h$; and $h_{0}$ is the initial thickness of the gap.

These equations have been presented by Murphy et al. (1986) to obtain an undrained saturated modulus. Thus, their boundary condition at the edge of the gap is the equation of fluid mass conservation between the gap and the annular pore. On the other hand, in the modified frame, only the intergranular gap is fully filled with the fluid, whereas the stiff (toroidal) pore is drained. Thus, in contrast to Murphy et al. (1986), our boundary condition (for equation 12) at the edge of the gap $(r=a)$ is that the fluid pressure $p$ is zero

$$
\left.p\right|_{r=a}=0 .
$$

Equation 12 is an inhomogeneous Bessel equation with a constant right-hand side. Substitution of

$$
p=q+\frac{C}{k^{2}}
$$

gives

$$
\frac{d^{2} q}{d r^{2}}+\frac{1}{r} \frac{d q}{d r}+k^{2} q=0
$$

which is a homogeneous Bessel equation of zero order (Abramowitz and Stegun, 1964). The general solution of this equation is

$$
q=C_{1} J_{0}(k r)
$$

which gives

$$
p=C_{1} J_{0}(k r)+\frac{C}{k^{2}}
$$

Substitution of this general solution into the boundary condition 14 gives

$$
C_{1}=-\frac{C}{k^{2} J_{0}(k a)},
$$

so that the pressure in the gap is given by

$$
p=\frac{C}{k^{2}}\left[1-\frac{J_{0}(k r)}{J_{0}(k a)}\right] \text {. }
$$

Then the force $\Delta F$ is

$$
\Delta F=2 \pi \int_{0}^{a} p(r) r d r=2 \pi \frac{C}{k^{2}}\left[\frac{a^{2}}{2}-\frac{1}{J_{0}(k a)} \int_{0}^{a} J_{0}(k r) r d r\right]
$$

or

$$
\Delta F=-\pi a^{2}\left[1-\frac{2 J_{1}(k a)}{k a J_{0}(k a)}\right] \frac{\Delta h K_{f}}{h_{0}} .
$$

Substitution of this force into equation 11 gives the following expression for the fluid-related gap stiffness: 


$$
K^{*}=\pi a^{2}\left[1-\frac{2 J_{1}(k a)}{k a J_{0}(k a)}\right] \frac{K_{f}}{h_{0}} .
$$

\section{Effective modulus of partially relaxed fluid}

In the low-frequency limit, $k \rightarrow 0$; thus, $K^{*}$ vanishes. This corresponds to the fact that at the low frequencies the fluid poses no resistance to gap deformation. At sufficiently low frequencies, the pressure in the gap will be equilibrated and thus will be zero throughout the gap. Conversely, in the limit of high frequency, equation 16 gives

$$
K^{*}=\frac{\pi a^{2}}{h_{0}} K_{f}
$$

This is the gap resistance in the unrelaxed state, when fluid has no time to escape from the gap within the half-period of the wave. Comparison of equations 16 and 17 shows that, at any given frequency, the gap stiffness is the same as the unrelaxed stiffness computed for a modified fluid with a bulk modulus

$$
K_{f}^{*}(P, \omega)=\left[1-\frac{2 J_{1}(k a)}{k a J_{0}(k a)}\right] K_{f} .
$$

Substitution of $K_{f}^{*}$ for the fluid modulus $K_{f}$ in equation 5 gives the final expression for the partially relaxed modulus $K_{m f}$ of the modified frame.

$$
\begin{aligned}
& \frac{1}{K_{m f}(P, \omega)} \\
& =\frac{1}{K_{h}}+\frac{1}{\frac{1}{K_{\mathrm{dry}}(P)}-\frac{1}{K_{h}}}+\frac{1}{\left(\frac{1}{K_{f}^{*}(P, \omega)}-\frac{1}{K_{g}}\right) \phi_{c}(P)} .
\end{aligned}
$$

Then, the corresponding partially relaxed shear modulus $\mu_{m f}$ of the modified frame can be obtained by substituting $K_{m f}$ for $K_{u f}$ in equation 4

$$
\frac{1}{\mu_{m f}(P, \omega)}=\frac{1}{\mu_{\mathrm{dry}}(P)}-\frac{4}{15}\left[\frac{1}{K_{\mathrm{dry}}(P)}-\frac{1}{K_{m f}(P, \omega)}\right] .
$$

Note that the wavenumber $k$ of the pressure wave, as given by equation 13 , is complex and frequency dependent

$$
k^{2}=-\frac{12 i \omega \eta}{h_{0}^{2} K_{f}}
$$

and so are the effective fluid modulus $K_{f}^{*}$ and partially relaxed frame moduli $K_{m f}$ and $\mu_{m f}$. This implies the presence of velocity dispersion and attenuation.

Equations 19 and 20 provide closed-form expressions for bulk and shear moduli of the modified frame (whereby soft pores are fluid-filled whereas stiff pores are dry). Once modified frame moduli are obtained, the moduli of the fully saturated medium can be obtained using Gassmann's or Biot's equations. If the frequency is small compared to Biot's characteristic frequency $f_{\text {Biot }}$, then the bulk modulus of the fully saturated medium can be computed using Gas- smann equation 9, whereas the shear modulus remains unchanged (see equation 10). If the frequency is comparable to or larger than $f_{\text {Biot }}$, then dispersion equations of the full Biot's theory of poroelasticity have to be used. This latter approach allows us to compute dispersion and attenuation due to both squirt and Biot's mechanisms.

\section{Asymptotes}

Frequency dependency of the partially relaxed modified frame moduli is controlled by the quantity

$$
k a=\frac{1}{\alpha}\left(-\frac{3 i \omega \eta}{K_{f}}\right)^{1 / 2}
$$

which depends on two new parameters: fluid viscosity $\eta$ and aspect ratio of the gaps (or compliant pores) $\alpha=h_{0} / 2 a$. Its squared value $\Omega=|k a|^{2}$ is proportional to frequency and can be used as dimensionless frequency. Figure $2 \mathrm{a}$ and $\mathrm{b}$ shows the predictions of the model based on equation 19 combined with Gassmann's equation 9 for the bulk modulus (real part) and inverse quality factor as functions of frequency at different pressure levels for the sample of Berea 5-600 sandstone (Han et al., 1986) fully saturated with water $\left(K_{f}\right.$ $\left.=2.2 \mathrm{GPa}, \rho_{f}=1031 \mathrm{~kg} / \mathrm{m}^{3}, \eta=10^{-3} \mathrm{~Pa} \cdot \mathrm{s}\right)$. Figure $3 \mathrm{a}$ and $\mathrm{b}$ shows the same dependencies for the same sample saturated with gas
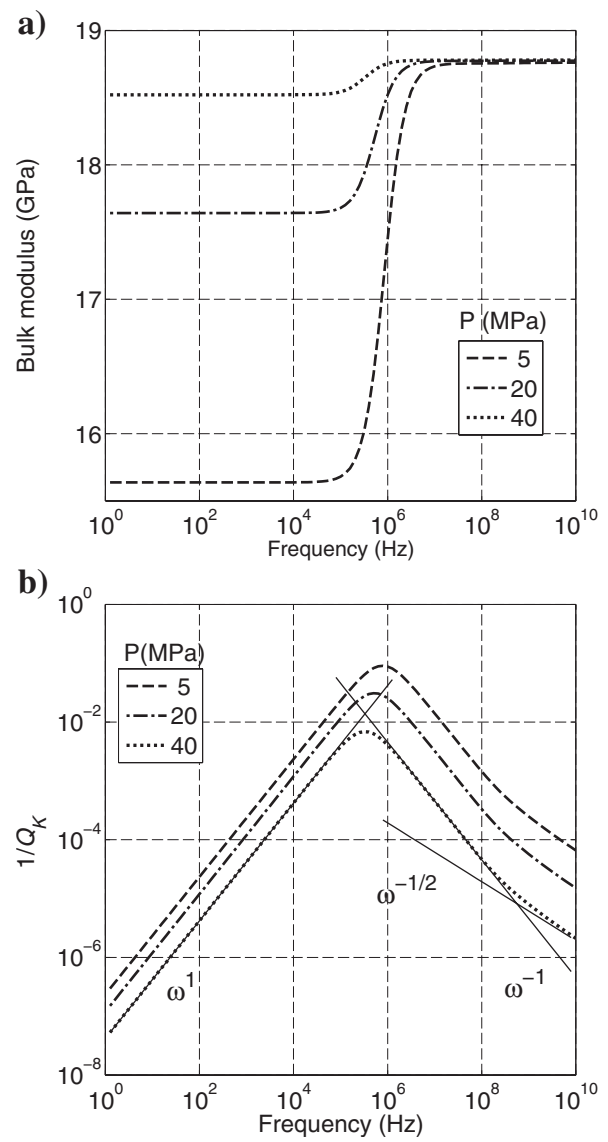

Figure 2. Predictions of (a) the real part of the bulk modulus and (b) dimensionless attenuation for a range of frequencies and pressures (shown by different line patterns) for a water-saturated sample of Berea 5-600 sandstone. Solid lines show asymptotic frequency dependencies of attenuation: $\omega^{1}$ at low frequencies, $\omega^{-1}$ at intermediate frequencies, and $\omega^{-1 / 2}$ at high frequencies. 
$\left(K_{f}=0.0022 \mathrm{GPa}, \rho_{f}=10.8 \mathrm{~kg} / \mathrm{m}^{3}, \quad \eta=11 \times 10^{-5} \mathrm{~Pa} \cdot \mathrm{s}\right)$. The workflow for estimation of $K_{h}$ and $\phi_{c}$ is described in Appendix A.

For the water-saturated rock (Figure $2 \mathrm{a}$ and $\mathrm{b}$ ), the bulk modulus shows a smooth transition from the low- to high-frequency limit. Similar behavior is observed for the gas-saturated rock (Figure 3a and b); however, not surprisingly the dispersion appears to be much smaller in this case. Attenuation for the water-saturated rock shows slightly more complex behavior, revealing existence of one additional asymptote at the intermediate frequencies (approximately $10^{6}-10^{8} \mathrm{~Hz}$ ), and one additional transition frequency (approximately $10^{9} \mathrm{~Hz}$ ).

To understand the behavior of attenuation in the water-saturated case, we consider three limiting cases.

1) Low frequencies. In this limit we have $|k a|^{2} \ll 1$ and

$$
|k a|^{2} \ll \frac{8 \phi_{c}(P)}{\left(\frac{1}{K_{\mathrm{dry}}(P)}-\frac{1}{K_{h}}\right) K_{f}} .
$$

Then, Taylor expansion of Bessel functions in equation 18 in powers of $k a$ gives
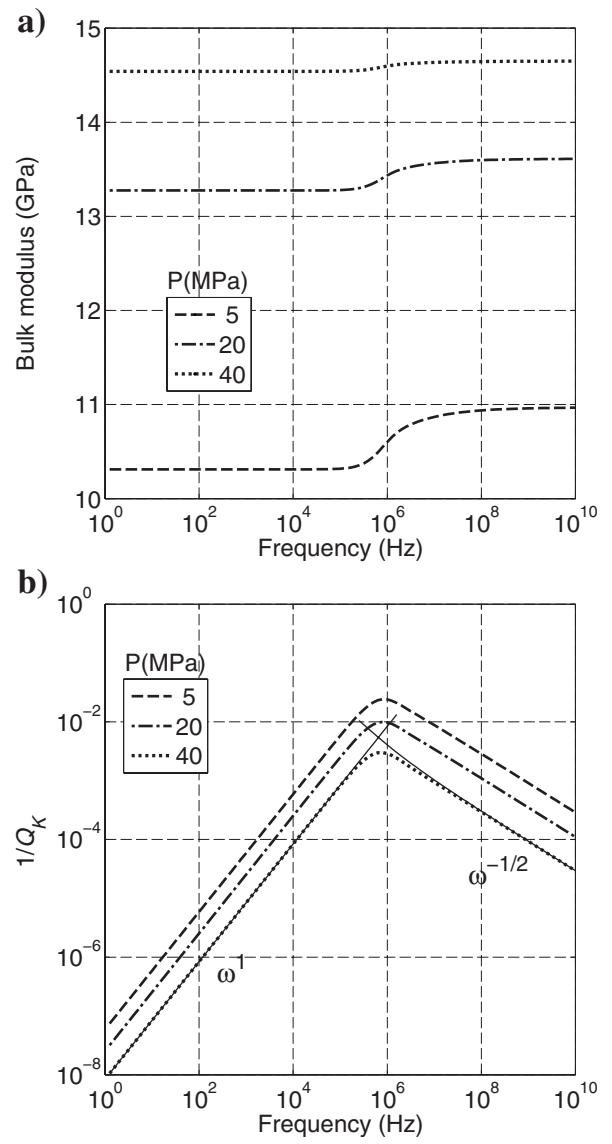

Figure 3. Predictions of (a) the real part of the bulk modulus and (b) dimensionless attenuation for a range of frequencies and pressures (shown by different line patterns) for a gas-saturated sample of Berea 5-600 sandstone. Solid lines show asymptotic frequency dependencies of attenuation: $\omega^{1}$ at low frequencies and $\omega^{-1 / 2}$ at high frequencies.

$$
K_{f}^{*}=-\frac{1}{8}(k a)^{2} K_{f}
$$

Substitution of this expression into equation 19 gives

$$
\frac{1}{K_{m f}(P, \omega)}=\frac{1}{K_{h}}+\frac{1}{\frac{1}{\frac{1}{K_{\text {dry }}(P)}-\frac{1}{K_{h}}}-\frac{(k a)^{2} K_{f}}{8 \phi_{c}(P)} .}
$$

Under condition 23 , equation 25 gives the following asymptote for the modified frame modulus:

$$
\begin{aligned}
K_{m f}(P, \omega)= & K_{d r y}(P) \\
& \times\left[1-\frac{(k a)^{2}}{8 \phi_{c}(P)}\left(\frac{1}{K_{d r y}(P)}-\frac{1}{K_{h}}\right)^{2} K_{f} K_{d r y}(P)\right] .
\end{aligned}
$$

This shows that in the low-frequency limit, the modified frame modulus tends to the dry modulus. The corresponding asymptotic expression for the dimensionless attenuation is

$$
\begin{aligned}
Q^{-1}(P, \omega) & =\frac{1}{8}\left(\frac{1}{K_{d r y}(P)}-\frac{1}{K_{h}}\right)^{2} \frac{K_{\mathrm{dry}}(P) K_{f}}{\phi_{c}(P)}|k a|^{2} \\
& =\frac{3}{8}\left(\frac{1}{K_{\mathrm{dry}}(P)}-\frac{1}{K_{h}}\right)^{2} \frac{\omega \eta K_{\mathrm{dry}}(P)}{\alpha^{2} \phi_{c}(P)}
\end{aligned}
$$

2) Intermediate frequencies.

$$
\frac{8 \phi_{c}(P)}{\left(\frac{1}{K_{\text {dry }}(P)}-\frac{1}{K_{h}}\right) K_{f}} \ll|k a|^{2} \ll 1 .
$$

In this case we can again use Taylor expansions 24 and 25. Under condition 28 , equation 25 reduces to

$$
K_{m f}(P, \omega)=K_{h}\left[1+\frac{8 \phi_{c}(P)}{(k a)^{2}} \frac{K_{h}}{K_{f}}\right] .
$$

The corresponding asymptotic expression for dimensionless attenuation is

$$
Q^{-1}(P, \omega)=\frac{8 \phi_{c}(P)}{|k a|^{2}} \frac{K_{h}}{K_{f}}=\frac{8 \phi_{c}(P) \alpha^{2} K_{h}}{3 \omega \eta} .
$$

Note that for this regime to exist we must have

$$
\frac{8 \phi_{c}(P)}{\left(\frac{1}{K_{\mathrm{dry}}(P)}-\frac{1}{K_{h}}\right)} \ll K_{f}
$$

This means that the fluid modulus must not be too small ("liquid case"). This explains why this regime is not observed in the gas case (see Figure 3b). Note that in this intermediate regime, attenuation is proportional to $1 / \omega$.

3) High frequencies: $|k a|^{2} \gg 1$. In this case equation 18 gives

$$
K_{f}^{*}=\left[1+\frac{2 i}{k a}\right] K_{f} .
$$


Substitution of expression 32 into equation 19 gives high-frequency asymptotes for $K_{\mathrm{mf}}$ and the corresponding attenuation factor. In particular, for the liquid case (condition 31), the expressions for these asymptotes are given by

$$
\begin{aligned}
K_{m f}(P, \omega)= & K_{u f}(P)+\phi_{c}(P) \frac{K_{h}^{2}}{K_{f}}\left(\frac{2 i}{k a}\right) \\
& \approx K_{h}\left[1+\frac{K_{h}}{K_{f}}\left(\frac{2 i \phi_{c}(P)}{k a}\right)\right]
\end{aligned}
$$

and

$$
Q^{-1}(P, \omega)=\frac{K_{h}}{K_{f}}\left(\frac{\sqrt{2} \phi_{c}(P)}{|k a|}\right)=\frac{\alpha \phi_{c}(P) K_{h}}{\left(3 \omega \eta K_{f} / 2\right)^{1 / 2}} .
$$

Note that in this high-frequency limit, attenuation is proportional to $1 / \sqrt{\omega}$.

\section{Simplified model for the liquid case}

As we have seen in the previous section, for the most important case of liquid saturation, the attenuation exhibits three asymptotic regimes. However, only two regimes are visible in the velocity dispersion. This may look strange, but can be easily understood if we notice that the real parts of the modified frame moduli at intermediate and high frequencies are $K_{h}$ (high-pressure dry modulus) and $K_{u f}$ (unrelaxed modulus), respectively, as given by equations 29 and 33 . As discussed earlier, for liquid saturation, the difference between these moduli is usually negligible (see equation 3 and corresponding discussion). Thus, the transition of the real parts of the modified frame moduli from the intermediate - to high-frequency regimes is unnoticeable and can be ignored. Moreover, the same can be said about attenuation. Indeed, attenuation corresponding to the high-frequency regime is negligibly small (this can be understood from the fact that in the intermediate regime the attenuation decays as $1 / \omega$ and rapidly becomes very small). We thus can conclude that the transition from intermediate - to high-frequency regimes is unimportant, and for all practical purposes the behavior described by equation 19 is accurately approximated by much simpler equation 25 , which describes a single dispersion transition. Equation 25 can also be rewritten in the form

$$
\frac{1}{K_{m f}(P, \omega)}=\frac{1}{K_{h}}+\frac{1}{\frac{1}{\frac{1}{K_{\mathrm{dry}}(P)}-\frac{1}{K_{h}}}+\frac{3 i \omega \eta}{8 \phi_{c}(P) \alpha^{2}}}
$$

with the shear modulus given by equation 20. It is interesting that dispersion and attenuation behavior predicted by this model does not explicitly depend on fluid compressibility. However, we should keep in mind that the fluid compressibility must be sufficiently small (modulus large) to satisfy condition 31 .

Equations 25 and 35 describe a simple transition of the modified frame modulus from $K_{\text {dry }}$ to $K_{\mathrm{h}}$. The characteristic transition frequency can be obtained from the intersection of asymptotes given by equations 27 and 30 . This gives

$$
\Omega_{t}=|k a|^{2}=\frac{8 \phi_{c}(P)}{K_{f}}\left[\frac{1}{K_{\mathrm{dry}}(P)}-\frac{1}{K_{h}}\right]^{-1}\left[\frac{K_{h}}{K_{\mathrm{dry}}(P)}\right]^{1 / 2} .
$$

In this equation, compliant porosity $\phi_{c}$ and dry modulus $K_{\text {dry }}$ are functions of pressure. Equation 36 can be simplified by noting that pressure variation of dry modulus is caused by progressive closure of compliant porosity. Indeed, according to Shapiro (2003), the difference between dry and high-stress compressibilities $K_{\mathrm{dry}}^{-1}-K_{h}^{-1}$ is proportional to the compliant porosity $\phi_{c}$

$$
\frac{1}{K_{\mathrm{dry}}(P)}-\frac{1}{K_{h}}=\frac{\phi_{c}(P)}{K_{h}} \theta_{c},
$$

where $\theta_{\mathrm{c}}$ is the parameter of stress sensitivity related to the compliance of compliant pores (or their effective aspect ratio). This gives

$$
\Omega_{t}=|k a|^{2}=\frac{8 K_{h}}{\theta_{c} K_{f}}\left[\frac{K_{h}}{K_{\text {dry }}(P)}\right]^{1 / 2}
$$

or

$$
\omega_{t}=\frac{8 \alpha^{2} K_{h}}{3 \eta \theta_{c}}\left[\frac{K_{h}}{K_{\mathrm{dry}}(P)}\right]^{1 / 2}
$$

For a small concentration of randomly oriented penny-shaped cracks, parameter $\theta_{c}$ can be related to their aspect ratio $\alpha$ by (see e.g., Mavko et al., 1998)

$$
\theta_{c}=\frac{K_{g}\left(3 K_{g}+4 \mu_{g}\right)}{\pi \alpha \mu_{g}\left(3 K_{g}+\mu_{g}\right)} .
$$

Using equation 40, expression 39 for squirt characteristic frequency can be written as

$$
\omega_{t}=\alpha^{3} \frac{B}{\eta}
$$

where

$$
B=\frac{8 \pi \mu_{h}\left(3 K_{h}+\mu_{h}\right)}{3\left(3 K_{h}+4 \mu_{h}\right)}\left[\frac{K_{h}}{K_{\mathrm{dry}}(P)}\right]^{1 / 2}
$$

is a combination of the dry moduli of the rock, which have dimensions of elastic moduli and are weakly dependent on pressure (in equation $42, \mu_{h}$ is dry shear modulus of the rock without compliant pores).

Note that in bilogarithmic scale, asymptotes 27 and 30 have identical slopes. Thus, the attenuation peak given by equation 25 is symmetric about the transition frequency $\Omega_{t}$, and maximum attenuation occurs exactly at $\Omega_{t}$ (or $\left.\omega_{t}\right)$. This maximum attenuation value can be obtained by substituting equation 38 into 25 and taking imaginary and real parts (note that $(k a)^{2}=-i|k a|^{2}$ ). This yields

$$
Q^{-1}\left(P, \omega_{t}\right)=\frac{K_{h}-K_{\mathrm{dry}}(P)}{K_{h}+K_{\mathrm{dry}}(P)}\left[\frac{K_{h}}{K_{\mathrm{dry}}(P)}\right]^{1 / 2} .
$$

Recall that $K_{h}$ is nothing more than $K_{\text {dry }}$ in the limit of high pressure. If the variation of dry bulk modulus with pressure is moderate, then $\left(K_{h} / K_{\mathrm{dry}}\right)^{1 / 2}=O(1)$ and thus the peak attenuation is equal to half of the fractional variation of the dry bulk modulus with pressure. For example, if the difference between $K_{\text {dry }}$ and $K_{h}$ is $20 \%$, then $Q$ will be approximately 10 . 
The theory presented here gives expressions for dispersion and attenuation in the modified frame (whereby soft pores are fluid-filled while stiff pores are dry). As mentioned earlier, the moduli of the fully saturated medium can be obtained using Gassmann's or Biot's equations.

\section{LABORATORY DATA EXAMPLES}

In this section, we illustrate predictions of our squirt model on several rocks and compare these predictions with laboratory measurements. Our aim is to illustrate the behavior of the model on a few samples. A comprehensive experimental validation of the model is certainly in order but will be done in a separate study.

Figure $4 \mathrm{a}$ and $\mathrm{b}$ shows a comparison between measured (solid circles) and predicted by our model (solid lines) compressional and shear velocities as functions of pressure for a water-saturated sample of Berea 5-600 sandstone (Han et al., 1986). We also plot the measurements carried out on a dry sample (empty circles) as well as the predictions by the Mavko-Jizba model (dashed lines) and Gassmann's (dotted lines) and Biot's equations (dash-dot lines). The parameters of the grain material are taken to be density $\rho_{\mathrm{g}}$ $=2653 \mathrm{~kg} / \mathrm{m}^{3}$ and the grain bulk modulus $K_{\mathrm{g}}=39 \mathrm{GPa}$, which is estimated by assuming that at the highest pressures the saturated bulk modulus is given by Gassmann's equation. The compliant po-
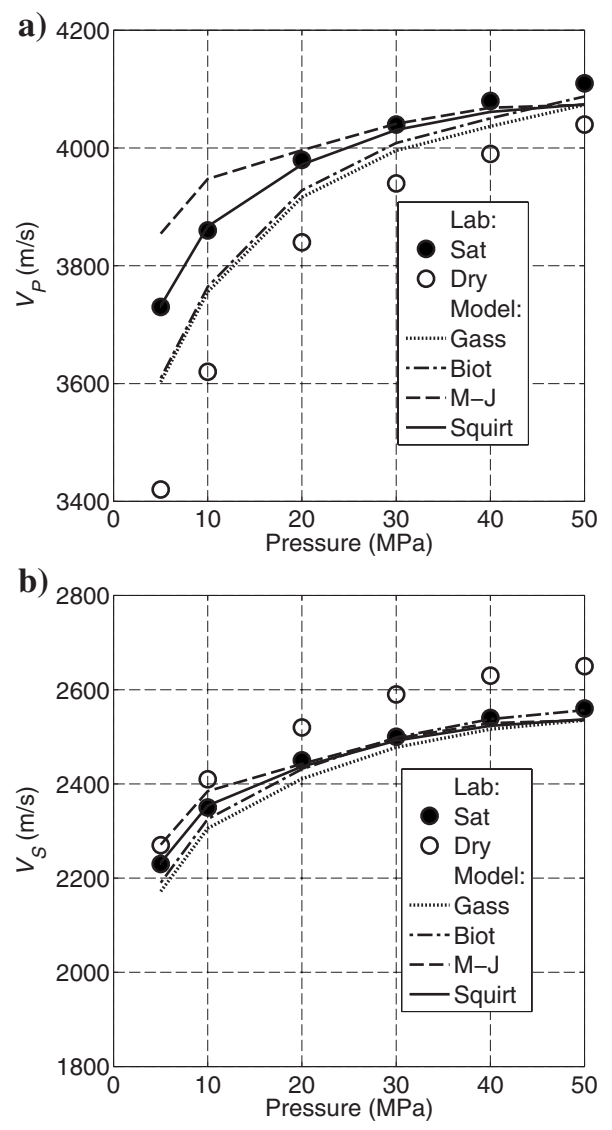

Figure 4. Velocities of (a) compressional and (b) shear waves in a sample of Berea 5-600 sandstone as a function of pressure: ultrasonic laboratory measurements on dry (empty circles) and water-saturated (solid circles) sample, predictions of the Mavko-Jizba (1991) model (dashed line), Gassmann's (dotted line) and Biot's (dash-dot line) theories, and predictions of the present model (solid line). rosity is obtained from velocity in the dry sample using the theory of Shapiro (2003). The aspect ratio of the grain contact $\alpha=0.01$ is obtained by the best fit of the predictions to the experimental data. The details of the parameter estimation are given in Appendix A. Figure $5 \mathrm{a}$ and $\mathrm{b}$ and Figure $6 \mathrm{a}$ and $\mathrm{b}$ show the dispersion and attenuation of compressional and shear velocities, respectively, as functions of pressure and frequency. We observe the decrease of dispersion and attenuation with increasing pressure. This is logical because pressure increase causes closure of compliant porosity.

Figure $7 \mathrm{a}$ and $\mathrm{b}$ shows a comparison between measured and predicted velocities as functions of pressure for a water-saturated carbonate sample S1 (Agersborg et al., 2008). The model parameters for the carbonate sample are taken to be $\rho_{g}=2670 \mathrm{~kg} / \mathrm{m}^{3}$ and $K_{g}$ $=82 \mathrm{GPa}$. The aspect ratio of the grain contacts obtained by the best fit of the prediction to the experimental data is $\alpha=0.01$. We see that for the sandstone and carbonate samples our model describes the observed shape of the pressure dependency reasonably well.

\section{DISCUSSION}

In this paper, we have developed a simple model of elastic wave attenuation and dispersion due to squirt flow between stiff and soft pores in a granular rock. The model applicability is shown on a cou-
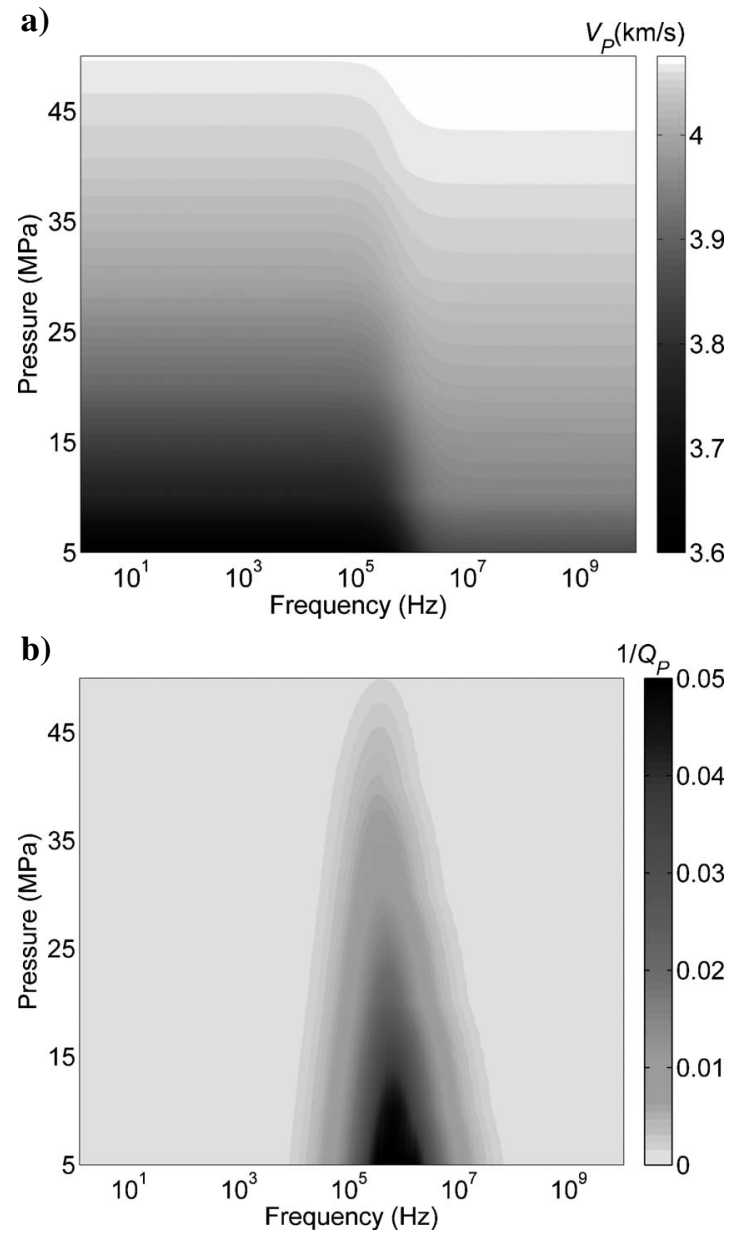

Figure 5. Predictions of (a) velocity and (b) attenuation of P-wave in a water-saturated sample of Berea 5-600 sandstone as a function of frequency and pressure. 
ple of illustrative examples. By construction, the model is exactly consistent with Gassmann's theory in the low-frequency limit and with the Mavko-Jizba model in the high-frequency limit. The expression for the characteristic frequency $\omega_{\mathrm{t}}$, equation 41 , is the same as the commonly used expression for squirt frequency (Jones, 1986), except that in our model the bulk modulus of the rock is replaced by a combination of bulk and shear moduli. Furthermore, for liquid-saturated rocks the attenuation and dispersion curves are symmetric about $\omega_{t}$ in $\log -\log$ scale. Attenuation $1 / Q$ is proportional to $\omega$ at low frequencies, and to $1 / \omega$ at high frequencies. The magnitude of attenuation and dispersion is directly related to the variation of dry bulk modulus with pressure. All of these features are also characteristic of the double-porosity model of Pride et al. (2004). The model presented here is designed to describe the same physical processes as the double-porosity model but uses a very different theoretical approach and is much simpler. An important advantage of our model is that it gives closed-form expressions for velocity and attenuation as functions of frequency and pressure. The model is particularly simple for the most important and rather general case of liquid-saturated rocks. In this case the dispersion and attenuation is given by equation 35 , which corresponds to a viscoelastic model known as a standard linear solid (Zener, 1948; Mavko et al., 1998). This makes the model easy to implement in viscoelastic and poroviscoelastic forward
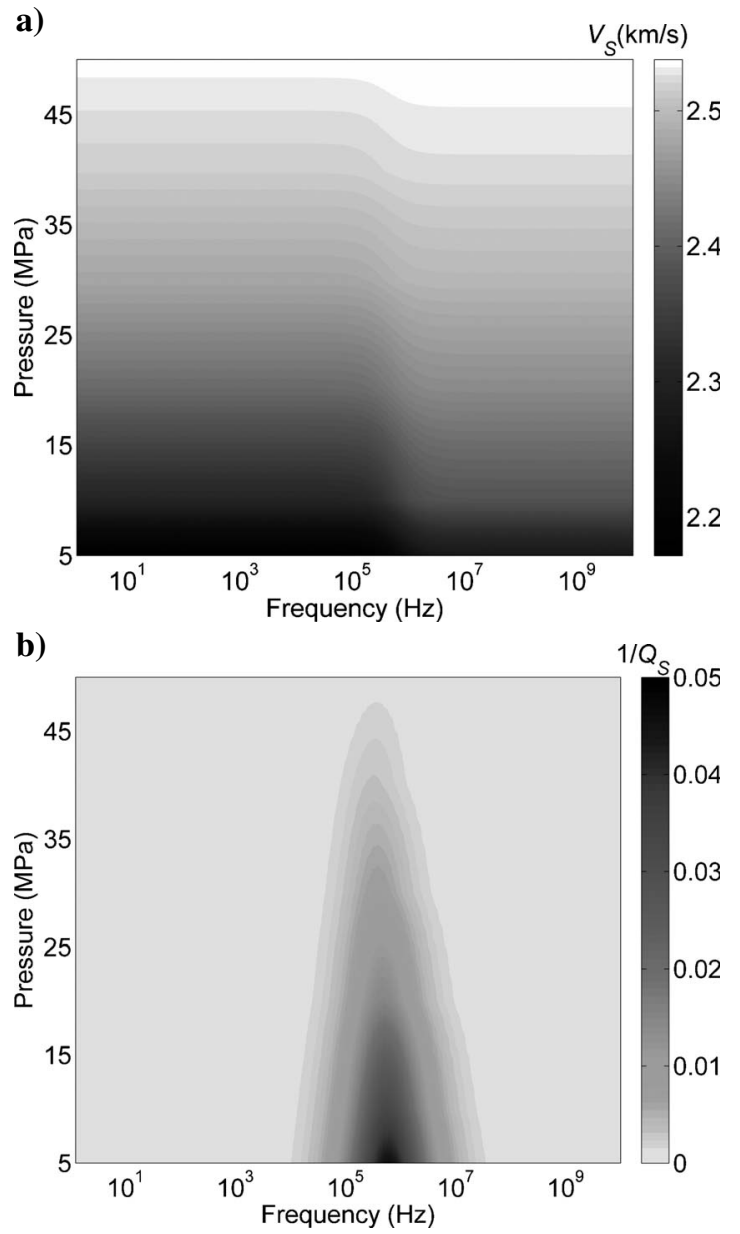

Figure 6. Predictions of (a) velocity and (b) attenuation of S-wave in a water-saturated sample of Berea 5-600 sandstone as a function of frequency and pressure. modeling using finite element and finite difference methods (Carcione, 2007).

The model contains one adjustable parameter - the aspect ratio of compliant pores. All other parameters can be measured or estimated from measurements of ultrasonic velocities and strains versus confining pressure on dry samples. The workflow for estimation of these parameters is described in Appendix A. One assumption in this workflow is that all compliant pores are closed at the upper limit of the pressure range of the measurements; hence, ultrasonic velocities become independent of pressure. Therefore, there is no squirt at these pressures and the saturated and dry velocities should approximately satisfy Gassmann's (or Biot's) equations. This is the case in the example given in Figure $4 \mathrm{a}$ and $\mathrm{b}$. However, in many other cases the compliant pores will not be completely closed at the pressure of 50 or even $100 \mathrm{MPa}$. This can be seen by the fact that in many cases the dry velocities at these pressures continue to increase with the pressure increase. This closure of remaining compliant porosity is responsible for the linear term in the velocity-pressure relationship of Eberhart-Phillips et al. (1989), which is based on the analysis of measurements on a large set of sandstone samples from different areas of the world (Han et al., 1986). The significance of this term has been recently demonstrated by Vernik and Hamman (2009) and Gurevich et al. (2009b). If the compliant pores close at higher pres-
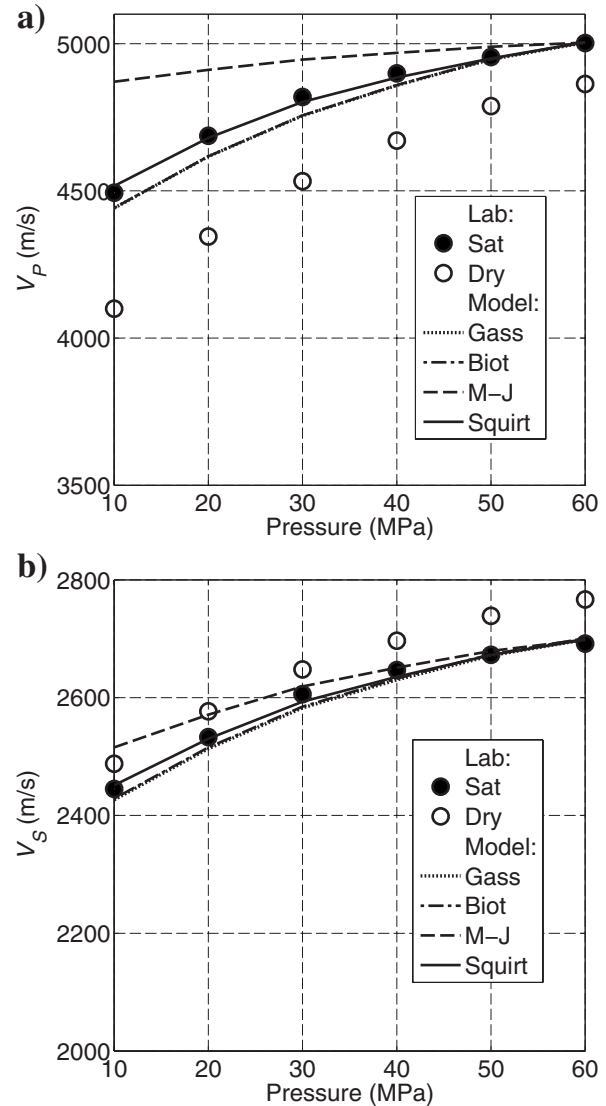

Figure 7. Velocities of (a) compressional and (b) shear waves in a sample of S1 carbonate as a function of pressure: ultrasonic laboratory measurements on dry (empty circles) and water-saturated (solid circles) sample, predictions of the Mavko-Jizba (1991) model (dashed line), Gassmann's (dotted line) and Biot's (dash-dot line) theories, and predictions of the model presented here (solid line). In this case, Gassmann's and Biot's predictions coincide. 
sures than the available range of experimental data, then the modulus $K_{h}$ cannot be estimated directly from the measurements. Instead, $K_{h}$ should be estimated using some effective medium theory assuming a typical aspect ratio of stiff pores in a particular rock (see e.g., Xu and White, 1995).

The particular frequency dependency of velocity and attenuation described by our theoretical model is a consequence of using soft porosity with a single aspect ratio. Many experimental studies show much more gradual variation of $Q$ with frequency, or even constant $Q$. In the context of squirt flow models, such frequency dependency is often explained by assuming a broad distribution of aspect ratios (O'Connell and Budiansky, 1977). A broad distribution of aspect ratios is also often invoked to explain the exponential stress dependency of elastic moduli. However, recent analysis based on the theoretical model of Shapiro (2003) suggests that such stress dependencies can be explained by a combination of only two aspect ratios: one for stiff pores and one for soft pores (Pervukhina et al., 2010). As noted above, this analysis is based on the dependency of elastic moduli in the range of confining stress up to 50-70 MPa. One can speculate that the stress dependency of elastic moduli in a broader range of stresses may reveal the presence of a broader range of pore aspect ratios. This in turn can affect the frequency dependency of velocity and attenuation caused by squirt. This issue requires further investigation.

Experimental validation of our model requires a comparison of its predictions against measurements of fluid-saturated velocities and attenuation factors versus frequency and pressure. The frequency range of ultrasonic measurements is usually quite narrow (e.g., $0.25-1 \mathrm{MHz}$ ) which makes it difficult to observe the velocity dispersion. One way to overcome this difficulty is to look at variations of velocity (and attenuation) with fluid viscosity. This can be done by saturating the same sample with several different fluids (e.g., Best and McCann, 1995; Adam et al., 2009) or using a fluid (e.g., glycerol) for which the viscosity can be varied by changing temperature (Jones, 1986). Alternatively, frequency dependency can be obtained from resonant bar (Born, 1941; Gardner, 1962; Wyllie et al., 1962; McCann and Sothcott, 2009) or forced-oscillation measurements (Batzle et al., 2006; Adam et al., 2009). A comprehensive comparison of model predictions with laboratory data will be a subject of a separate study.

\section{CONCLUSIONS}

We have developed a simple model of squirt-flow relaxation in granular fluid-saturated media. The model gives closed-form expressions for velocity and attenuation as functions of frequency and pressure. The results are exactly consistent with Gassmann's theory in the low-frequency limit and with Mavko-Jizba unrelaxed moduli in the high-frequency limit. For liquid-saturated rocks, the attenuation and dispersion correspond to the model of standard linear solid, with coefficients directly related to the elastic and hydraulic properties of the medium. Attenuation factor $1 / Q$ is proportional to $\omega$ at low frequencies and to $1 / \omega$ at high frequencies. The magnitude of attenuation and dispersion is directly related to the variation of the dry bulk modulus with pressure and is relatively independent of fluid properties. The model contains one adjustable parameter - the aspect ratio of compliant pores (grain contacts). All other parameters can be measured or estimated from measurements of ultrasonic velocities and strains versus confining pressure on dry samples.

\section{ACKNOWLEDGMENTS}

We gratefully acknowledge financial support of the Australian Research Council (Discovery-Project DP1096232) and the sponsors of the Curtin Reservoir Geophysics Consortium (CRGC). DM was supported by the Australian Postgraduate Award and CSIRO scholarship. OP is grateful to Petrobras for PhD support at Curtin University. We also thank Colin M. Sayers and Serge A. Shapiro for stimulating discussions.

\section{APPENDIX A}

\section{ESTIMATION OF MODEL PARAMETERS}

Our model given by equations 35 and 20 predicts modified frame moduli and, when combined with Gassmann's or Biot's theory, compressional and shear wave moduli, velocities, and attenuation coefficients of a fully saturated rock as a function of frequency and material properties (of the rock frame and pore fluid). Because the model contains several parameters, it is always possible to fit it to data sufficiently well by varying the unknown parameters. It is therefore critical to measure or estimate independently as many parameters as possible (i.e., to perform a controlled experiment). In a typical laboratory setup, compressional and shear ultrasonic velocities are measured on dry and fluid-saturated rock samples as a function of pressure. Thus, parameters $K_{\text {dry }}$ and $\mu_{\text {dry }}$ as functions of pressure can be obtained from the ultrasonic measurements using standard equations $K_{\text {dry }}=\left[V_{\mathrm{P}}^{2}-(4 / 3) V_{\mathrm{S}}^{2}\right] \rho$ and $\mu_{\text {dry }}=V_{\mathrm{S}}^{2} \rho$. Below we describe how the other parameters of the model can be estimated from the laboratory measurements.

\section{High-pressure modulus}

High-pressure modulus $K_{h}$ can be taken as $K_{\text {dry }}$ at the highest pressure available. Note that this approach assumes that at this pressure all of the compliant porosity is closed and corresponds to the fact that $K_{\text {dry }}$ as function of pressure has leveled off at this pressure value.

\section{Compliant porosity}

Compliant porosity $\phi_{c}$ cannot be directly measured; however, it can be estimated from the variation of total porosity with pressure. For instance, this variation can be estimated by measuring volumetric strain as a function of pressure using strain gauges. Once the total porosity variation is known, stiff porosity can be estimated by fitting a linear trend to total porosity in the high-pressure range where compliant porosity is assumed closed (and thus total porosity equals stiff porosity) (Figure A-1). Compliant porosity can be obtained as a difference between total porosity and the linear trend of stiff porosity extrapolated to the lower pressures (Walsh, 1965; Mavko and Jizba, 1991; Pervukhina et al., 2010). However, note that because compliant porosities are usually 0.001 or less, high-accuracy measurements of total porosity variations with pressure are required.

If such precise porosity measurements are not available, compliant porosity can be estimated from the stress dependency of dry elastic moduli obtained from ultrasonic measurements as suggested by Pervukhina et al. (2010). Stress dependency of the dry bulk modulus can be approximated as follows (Shapiro, 2003)

$$
1 / K_{\mathrm{dry}}(P)-1 / K_{h}=\left(\phi_{c 0} / P_{h}\right) \exp \left(-P / P_{h}\right),
$$

where $\phi_{c 0}$ is compliant porosity at zero pressure and $P_{h}$ is a characteristic pressure at which compliant porosity closes. The parameters 


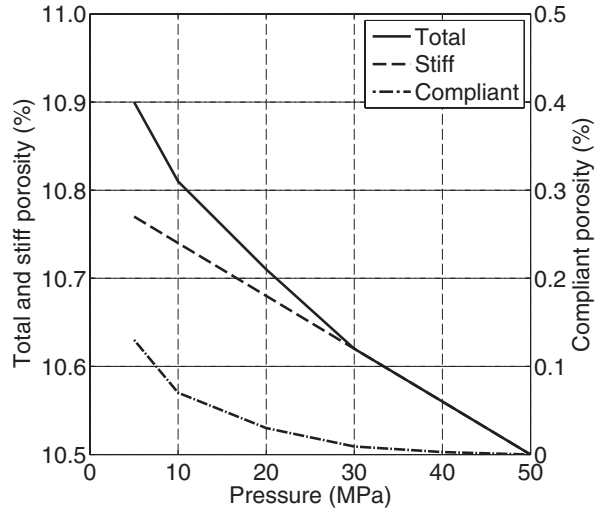

Figure A-1. Porosity versus confining pressure. Compliant porosity (dash-dot line) at a given pressure is estimated as the difference between the total porosity (solid line) and the extrapolation of the highpressure porosity versus pressure trend (dashed line).

$\phi_{c 0} / P_{h}$ and $P_{h}$ can be obtained by fitting of the stress dependency of bulk moduli using equation 44 and $\phi_{c 0}$ can be estimated as a ratio of these fitting parameters. Then the pressure variation of compliant porosity can be written as

$$
\phi_{c}(P)=\phi_{c 0} \exp \left[P / P_{h}\right] .
$$

Note that neither of the two compliant porosity estimation approaches utilizes the velocity or attenuation measurements on saturated samples. Therefore, these estimates are independent of dispersion/attenuation data.

\section{Aspect ratio of the contact gap}

Like compliant porosity, aspect ratio $\alpha$ of the grain contact gap cannot be directly measured. It is tempting to estimate the gap aspect ratio from the variation of, say, dry moduli with compliant porosity (e.g., using elastic effective medium theory; Küster and Toksöz, 1974; Berryman, 1980). However, the aspect ratio that controls fluid pressure relaxation in the flat intergranular gap may not be related to the aspect ratio of penny-shaped cracks that control the pressure variation of the effective elastic moduli of the dry rock. In this study, we use gap aspect ratio $\alpha$ as a free-fitting parameter and estimate it as the value that provides the best fit for modulus-pressure dependency on saturated samples (Figures 4 and 7).

\section{REFERENCES}

Abramowitz, M., and I. A. Stegun, 1964, Handbook of mathematical functions: National Bureau of Standards Applied Mathematics Series.

Adam, L., M. L. Batzle, K. T. Lewallen, and K. van Wijk, 2009, Seismic wave attenuation in carbonates: Journal of Geophysical Research, 114, B06208.

Agersborg, R., T. A. Johansen, M. Jakobsen, J. Sothcott, and A. Best, 2008 Effects of fluids and dual-pore systems on pressure-dependent velocities and attenuations in carbonates: Geophysics, 73, no. 5, N35-N47.

Auriault, J.-L., and C. Boutin, 1994, Deformable porous media with double porosity III, Acoustics: Transport in Porous Media, 14, 143-162.

Batzle, M. L., D.-H. Han, and R. Hofmann, 2006, Fluid mobility and frequency-dependent seismic velocity — Direct measurements: Geophysics, 71, no. 1, N1-N9.

Berryman, J. G., 1980, Long-wavelength propagation in composite elastic media - I. Spherical inclusions, II. Ellipsoidal inclusions: Journal of the Acoustical Society of America, 68, 1809-1831.

- 2007, Seismic waves in rocks with fluids and fractures: Geophysical Journal International, 171, 954-974

Best, A., and C. McCann, 1995, Seismic attenuation and pore-fluid viscosity in clay-rich reservoir sandstones: Geophysics, 60, 1386-1397.
Biot, M. A., 1956a, Theory of propagation of elastic waves in a fluid-saturated porous solid. I. Low-frequency range: Journal of the Acoustical Society of America, 28, 168-178.

- 1956b, Theory of propagation of elastic waves in a fluid-saturated porous solid. II. Higher frequency range: Journal of the Acoustical Society of America, 28, 179-191.

1962, Mechanics of deformation and acoustic propagation in porous media: Journal of Applied Physics, 33, 1482-1498.

Born, W. T., 1941, The attenuation constant of earth materials: Geophysics, 6, 132-148.

Bourbié, T., O. Coussy, and B. Zinszner, 1987, Acoustics of porous media: Technip.

Carcione, J. M., 2007, Wave fields in real media. Theory and numerical simulation of wave propagation in anisotropic, anelastic, porous and electromagnetic media: Elsevier Science.

Chapman, M., S. V. Zatsepin, and S. Crampin, 2002, Derivation of a microstructural poroelastic model: Geophysical Journal International, 151, 427-451.

Digby, P. J., 1982, The effective elastic moduli of porous granular rocks Journal of Applied Mechanics ASME, 48, 803-808.

Dutta, N. C., and H. Ode, 1979a, Attenuation and dispersion of compressional waves in fluid-filled porous rocks with partial gas saturation (White model) - Part I, Biot theory: Geophysics, 44, 1777-1788.

$-1979 \mathrm{~b}$, Attenuation and dispersion of compressional waves in fluidfilled porous rocks with partial gas saturation (White model) — Part II: results: Geophysics, 44, 1789-1805.

Dvorkin, J., G. Mavko, and A. Nur, 1995, Squirt flow in fully saturated rocks: Geophysics, 60, 97-107.

Eberhart-Phillips, D., D. -H. Han, and M. D. Zoback, 1989, Empirical relationships among seismic velocity, effective pressure, porosity and clay content in sandstone: Geophysics, 54, 82-89.

Endres, A. L., and R. J. Knight, 1997, Incorporating pore geometry and fluid pressure communication into modeling the elastic behavior of porous rocks: Geophysics, 62, 106-117.

Gardner, G. H. F., 1962, Extensional waves in fluid-saturated porous cylinders: Journal of the Acoustical Society of America, 34, 36-40.

Gassmann, F., 1951, Über die Elastizität poröser Medien. Viertel Naturforsch Ges Zürich, 96, 1-23.

Gist, G. A., 1994, Interpreting laboratory velocity measurements in partially gas-saturated rocks: Geophysics, 59, 1100-1109.

Grechka, V., 2009, Fluid-solid substitution in rocks with disconnected and partially connected porosity: Geophysics, 74, no. 5, WB89-WB95.

Gurevich, B., and S. L. Lopatnikov, 1995, Velocity and attenuation of elastic waves in finely layered porous rocks: Geophysical Journal International, 121,933-947.

Gurevich, B., D. Makarynska, and M. Pervukhina, 2009a, Ultrasonic modul for fluid-saturated rocks: Mavko-Jizba relations rederived and generalized: Geophysics, 74, no. 4, N25-N30.

, 2009b, Are penny-shaped cracks a good model for compliant porosity? 79th Annual International Meeting, SEG, Expanded Abstracts, 3431-3435.

Han, D.-H., A. Nur, and D. Morgan, 1986, Effects of velocity and clay content on wave velocities in sandstones: Geophysics, 51, 2093-2010.

Johnson, D. L., 2001, Theory of frequency dependent acoustics in patchysaturated porous media: Journal of the Acoustical Society of America, 110, 682-694.

Jones, T., 1986, Pore fluids and frequency dependent wave propagation in rocks: Geophysics, 51, 1939-1953.

Küster, G. T., and M. N. Toksöz, 1974, Velocity and attenuation of seismic waves in two-phase media - Part I: Theoretical formulations: Geophysics, 39, 587-606.

Lopatnikov, S. L., P. Y. Gorbachev, and B. Gurevich, 1990, Acoustic wave propagation in a porous medium with randomly non-homogeneous gas distribution: Izvestija Academy of Sciences USSR, Physics of the Solid Earth, 26, 468-471.

Lopatnikov, S. L., and B. Gurevich, 1988, Transformational mechanism of elastic wave attenuation in saturated porous media: Izvestija Academy of Sciences USSR, Physics of the Solid Earth, 24, 151-154.

Mavko, G., and D. Jizba, 1991, Estimating grain-scale fluid effects on velocity dispersion in rocks: Geophysics, 56, 1940-1949.

_ 1994, The relation between seismic P-and S-wave velocity dispersion in saturated rocks: Geophysics, 59, 87-92.

Mavko, G., and A. Nur, 1975, Melt squirt in the aesthenosphere: Journal of Geophysical Research, 80, 1444-1448.

-1979 , Wave attenuation in partially saturated rocks: Geophysics, 44 $161-178$.

Mavko, G., T. Mukerji, and J. Dvorkin, 1998, The rock physics handbook: Tools for seismic analysis in porous media: Cambridge University Press.

Mayr, S. I., and H. Burkhardt, 2006, Ultrasonic properties of sedimentary rocks: effect of pressure, saturation, frequency and microcracks: Geophysical Journal International, 164, 246-258.

McCann, C., and J. Sothcott, 2009, Sonic to ultrasonic Q of sandstones and 
limestones, laboratory measurements at in situ pressures: Geophysics, 74, no. 2, WA93-WA101.

Müller, T. M., and B. Gurevich, 2005, Wave-induced fluid flow in random porous media: attenuation and dispersion of elastic waves: Journal of the Acoustical Society of America, 117, 2732-2741.

Murphy, W. F., K. W. Winkler, and R. L. Kleinberg, 1986, Acoustic relaxation in sedimentary rocks, dependence on grain contacts and fluid saturation: Geophysics, 51, 757-766.

O'Connell, R., and B. Budiansky, 1977, Viscoelastic properties of fluid-saturated cracked solids: Journal of Geophysical Research, 82, 5719-5735.

Palmer, I. D., and M. L. Traviolia, 1980, Attenuation by squirt flow in undersaturated gas sands: Geophysics, 45, 1780-1792.

Pervukhina, M., B. Gurevich, D. N. Dewhurst, and A. F. Siggins, 2010, Applicability of velocity-stress relationships based on the dual porosity concept to isotropic porous rocks: Geophysical Journal International, doi: 10.1111/j.1365-246X.2010.04535.x.

Pride, S. R., and J. G. Berryman, 2003, Linear dynamics of double-porosity dual-permeability materials. I. Governing equations and acoustic attenuation: Physical Review E, 68, 036603.

Pride, S. R., J. G. Berryman, and J. M. Harris, 2004, Seismic attenuation due to wave-induced flow: Journal of Geophysical Research, 109, B01201.

Pride, S. R., J. M. Harris, D. L. Johnson, A. Mateeva, K. T. Nihel, R. L. Nowack, J. W. Rector, H. Spetzler, R. Wu, T. Yamomoto, J. G. Berryman, and M. Fehler, 2003, Permeability dependence of seismic amplitudes: The Leading Edge, 22, 518-525.

Sayers, C. M., and D.-H. Han, 2002, The effect of pore fluid on the stress-dependent elastic wave velocities in sandstones: 72nd Annual International Meeting, SEG, Expanded Abstracts, 1842-1845.

Sayers, C. M., and M. Kachanov, 1995, Microcrack-induced elastic wave anisotropy of brittle rocks: Journal of Geophysical Research, 100, 4149-4156.
Shapiro, S. A., 2003, Elastic piezosensitivity of porous and fractured rocks: Geophysics, 68, 482-486.

Thomsen, L., 1985, Biot-consistent elastic moduli of porous rocks: Low-frequency limit: Geophysics, 50, 2797-2807.

Toms, J., T. M. Müller, and B. Gurevich, 2007, Seismic attenuation in porous rocks with random patchy saturation: Geophysical Prospecting, 55, 671-678.

Vernik, L., and J. Hamman, 2009, Stress sensitivity of sandstones and 4D applications: The Leading Edge, 28, 90-93.

Walsh, J. B., 1965, The effect of cracks on the compressibility of rock: Journal of Geophysical Research, 70,381-389.

White, J. E., 1975, Computed seismic speeds and attenuation in rocks with partial gas saturation: Geophysics, 40, 224-232.

White, J. E., 1983, Underground sound: Application of seismic waves: Elsevier Science.

White, J. E., N. G. Mikhaylova, and F. M. Lyakhovitsky, 1975, Low frequency seismic waves in fluid saturated layered rocks: Izvestija Academy of Sciences USSR, Physics of the Solid Earth, 11, 654-659.

Winkler, K. W., 1983, Contact stiffness in granular porous materials: comparison between theory and experiment: Geophysical Research Letters, 10, 1073-1076.

Wulff, A.-M.., and H. Burkhardt, 1997, Mechanisms affecting ultrasonic wave propagation in fluid-containing sandstones under high hydrostatic pressure: Journal of Geophysical Research, 102, 3043-3050.

Wyllie, M. R., G. H. F. Gardner, and A. R. Gregory, 1962, Studies of elastic wave attenuation in porous media: Geophysics, 27, 569-589.

Xu, S., and R. E. White, 1995, A new velocity model for clay-sand mixtures: Geophysical Prospecting, 43, 91-118.

Zener, C., 1948, Elasticity and anelasticity of metals: University of Chicago Press. 